\title{
Serum neurofilament light chain protein is a measure of disease intensity in frontotemporal dementia OPEN
}

Jonathan D. Rohrer, PhD Ione O.C. Woollacott, MRCP

Katrina M. Dick, BSc Emilie Brotherhood, BSc Elizabeth Gordon, MSc Alexander Fellows, BSc Jamie Toombs, MSc Ronald Druyeh, BSc M. Jorge Cardoso, PhD Sebastien Ourselin, PhD Jennifer M. Nicholas, $\mathrm{PhD}$

Niklas Norgren, PhD Simon Mead, PhD Ulf Andreasson, PhD Kaj Blennow, PhD Jonathan M. Schott, MD Nick C. Fox, MD Jason D. Warren, PhD Henrik Zetterberg, PhD

Correspondence to Dr Rohrer: j.rohrer@ucl.ac.uk

\section{ABSTRACT}

Objective: To investigate serum neurofilament light chain ( $\mathrm{NfL}$ ) concentrations in frontotemporal dementia (FTD) and to see whether they are associated with the severity of disease.

Methods: Serum samples were collected from 74 participants (34 with behavioral variant FTD [bvFTD], 3 with FTD and motor neuron disease and 37 with primary progressive aphasia [PPA]) and 28 healthy controls. Twenty-four of the FTD participants carried a pathogenic mutation in C9orf72 (9), microtubule-associated protein tau (MAPT; 11), or progranulin (GRN; 4). Serum NfL concentrations were determined with the NF-Light kit transferred onto the single-molecule array platform and compared between FTD and healthy controls and between the FTD clinical and genetic subtypes. We also assessed the relationship between NfL concentrations and measures of cognition and brain volume.

Results: Serum NfL concentrations were higher in patients with FTD overall (mean $77.9 \mathrm{pg} / \mathrm{mL}$ [SD $51.3 \mathrm{pg} / \mathrm{mL}$ ]) than controls $(19.6 \mathrm{pg} / \mathrm{mL}$ [SD $8.2 \mathrm{pg} / \mathrm{mL}$; $p<0.001)$. Concentrations were also significantly higher in bvFTD $(57.8 \mathrm{pg} / \mathrm{mL}$ [SD $33.1 \mathrm{pg} / \mathrm{mL}])$ and both the semantic and nonfluent variants of PPA (95.9 and $82.5 \mathrm{pg} / \mathrm{mL}$ [SD 33.0 and $33.8 \mathrm{pg} / \mathrm{mL}$ ], respectively) compared with controls and in semantic variant PPA compared with logopenic variant PPA. Concentrations were significantly higher than controls in both the C9orf72 and MAPT subgroups (79.2 and 40.5 pg/mL [SD 48.2 and $20.9 \mathrm{pg} / \mathrm{mL}$ ], respectively) with a trend to a higher level in the GRN subgroup (138.5 pg/mL [SD $103.3 \mathrm{pg} / \mathrm{mL}$ ). However, there was variability within all groups. Serum concentrations correlated particularly with frontal lobe atrophy rate ( $r=0.53, p=0.003$ ).

Conclusions: Increased serum NfL concentrations are seen in FTD but show wide variability within each clinical and genetic group. Higher concentrations may reflect the intensity of the disease in FTD and are associated with more rapid atrophy of the frontal lobes. Neurology ${ }^{\circledR} 2016 ; 87: 1329-1336$

\section{GLOSSARY}

bvFTD = behavioral variant frontotemporal dementia; FTD $=$ frontotemporal dementia; GENFI $=$ Genetic Frontotemporal Dementia Initiative; IvPPA = logopenic variant of primary progressive aphasia; MND = motor neuron disease; $\mathbf{N f L}=$ neurofilament light chain; $\mathbf{n f v P P A}=$ nonfluent variant of primary progressive aphasia; PPA = primary progressive aphasia; PPA-NOS = primary progressive aphasia not otherwise specified; Simoa = single-molecule array; svPPA = semantic variant of primary progressive aphasia.

Frontotemporal dementia (FTD) is a common cause of early-onset dementia. ${ }^{1}$ Clinically, patients present with either changes in personality (behavioral variant FTD [bvFTD]) or impaired language (primary progressive aphasia [PPA]), although overlap with motor neuron disease (FTD-MND) is not uncommon. ${ }^{1}$ FTD has an autosomal dominant genetic cause in around a quarter of people, with mutations in the progranulin $(G R N)$, chromosome 9 open reading frame 72 (C9orf72), and microtubule-associated protein tau (MAPT) genes being commonest. ${ }^{2}$

Few fluid biomarkers have been investigated in FTD, although there have now been a number of studies of neurofilament concentration in the CSF. ${ }^{3-11}$ Higher neurofilament light chain

From the Dementia Research Centre (J.D.R., I.O.C.W., K.M.D., E.B., E.G., A.F., M.J.C., S.O., J.M.N., J.M.S., N.C.F., J.D.W.), MRC Prion Unit (S.M., R.D.), Department of Neurodegenerative Disease, and Department of Molecular Neuroscience (J.T., H.Z.), UCL Institute of Neurology, Queen Square; Centre for Medical Image Computing (J.M.C., S.O.), University College London; Department of Medical Statistics (J.M.N.), London School of Hygiene and Tropical Medicine, UK; UmanDiagnostics (N.N.), Umeå; and Clinical Neurochemistry Laboratory (U.A., K.B., H.Z.), Department of Psychiatry and Neurochemistry, Institute of Neuroscience and Physiology, Sahlgrenska Academy at the University of Gothenburg, Mölndal, Sweden. Go to Neurology.org for full disclosures. Funding information and disclosures deemed relevant by the authors, if any, are provided at the end of the article. The Article Processing Charge was paid by Medical Research Council.

This is an open access article distributed under the terms of the Creative Commons Attribution License 4.0 (CC BY), which permits unrestricted use, distribution, and reproduction in any medium, provided the original work is properly cited. 
(NfL) levels are believed to represent axonal degeneration, ${ }^{12,13}$ and while early studies showed variability in CSF concentrations in FTD,${ }^{4-10}$ a more recent study has suggested that CSF NfL levels correlate with disease severity. ${ }^{11}$

There is considerable interest in developing blood-based biomarkers because of their convenience and higher acceptability relative to CSF. NfL can be measured in serum with standard immunoassay formats, ${ }^{14}$ but those based on ELISA or electrochemiluminescence methods lack the analytical sensitivity to measure low levels. For this reason, we developed an immunoassay based on the single-molecule array (Simoa) technique $^{15}$ that allows quantification down to subfemtomolar concentrations $(<1 \mathrm{pg} / \mathrm{mL})$ of the analyte and is 25-fold more sensitive than the previous electrochemiluminescence-based method. ${ }^{16}$ Using this assay, we aimed to investigate serum NfL concentrations in FTD. Our hypotheses were that serum NfL concentration would be elevated in patients with FTD compared with healthy controls, that concentrations would vary between FTD subgroups, and that increased serum NfL levels would reflect the disease intensity or rate of progression.

\footnotetext{
METHODS Seventy-four participants were consecutively recruited from the University College London FTD study: 34 participants with bvFTD according to Rascovsky criteria, ${ }^{17} 3$ participants with FTD-MND, ${ }^{18}$ and 37 participants with PPA according to the Gorno-Tempini criteria. ${ }^{19}$ Of the 37 PPA participants, 13 had the nonfluent variant (nfvPPA), 10 had the semantic variant (svPPA), 7 had the logopenic variant (lvPP), and 7 did not fit criteria for any of the 3 variants (PPA-NOS, not otherwise specified). We did not include patients fulfilling criteria for lvPPA in the overall FTD analysis because they are likely to have underlying Alzheimer disease pathologically. ${ }^{1,2}$ Data were compared with data from 28 healthy control participants matched for age and sex who had been collected as part of a study of neurodegenerative disease (table 1). Twenty-four of
}

the FTD participants carried a pathogenic mutation: 9 with an expansion in C9orf72 (8 with bvFTD, 1 with nfvPPA), 11 with an $M A P T$ mutation (all with bvFTD), and 4 with a $G R N$ mutation (1 with bvFTD, 1 with nfvPPA, and 2 with PPA-NOS). No mutations were found in the other participants. No significant differences were noted in age or sex between any of the groups, and no significant difference in disease duration was seen between the clinical or genetic FTD subgroups.

Standard protocol approvals, registrations, and patient consents. Approval for the study was obtained from the local ethics committee, and all participants provided written consent to take part.

Measurement of NfL concentrations. Serum samples were collected from each of the participants and then processed, divided into aliquots, and frozen at $-80^{\circ} \mathrm{C}$ according to standardized procedures. Serum NfL concentrations were measured with the NF-Light assay from UmanDiagnostics (Umeå, Sweden) and transferred onto the Simoa platform with a home-brew kit (Quanterix Corp, Boston, MA). Detailed instructions can be found in the Simoa Homebrew Assay Development Guide (Quanterix). In short, paramagnetic carboxylated beads (catalog no. 100451, Quanterix) were activated by adding 5\% (vol/vol) $10 \mathrm{mg} / \mathrm{mL}$ 1-ethyl-3-(3-dimethylaminopropyl) carbodiimide (catalog no. 100022, Quanterix) to a magnetic beads solution with $1.4 \times 10^{6}$ beads $/ \mu \mathrm{L}$. After a 30 -minute incubation at room temperature, the beads were washed with a magnetic separator, and an initial volume, i.e., 1-ethyl-3-(3-dimethylaminopropyl) carbodiimide + bead solution volume in the previous step, of $0.3 \mathrm{mg} / \mathrm{mL}$ ice cold solution of the capture antibody (UD1, UmanDiagnostics) was added. After a 2-hour incubation on a mixer (2,000 rpm, Multi-Tube Vortexer, Allsheng, China) at room temperature, the beads were washed, and an initial reaction volume of blocking solution was added. After 3 washes, the conjugated beads were suspended and stored at $4{ }^{\circ} \mathrm{C}$ pending analysis. Before analysis, the beads were diluted to 2,500 beads $/ \mu \mathrm{L}$ in bead diluent. The detection antibody $(1 \mathrm{mg} / \mathrm{mL}$, UD2, UmanDiagnostics) was biotinylated by adding $3 \%$ (vol/vol) $3.4 \mathrm{mmol} / \mathrm{L}$ EZ-Link NHS-PEG4-Biotin (Quanterix), followed by a 30 -minute incubation at room temperature. Free biotin was removed with spin filtration (Amicon Ultra-2, 50 $\mathrm{kDa}$, Sigma, St. Louis, MO), and the biotinylated antibody was stored at $4^{\circ} \mathrm{C}$ pending analysis. The serum samples were assayed in duplicate on a Simoa HD-1 instrument (Quanterix) using a 2-step assay dilution protocol that starts with an aspiration of the bead diluent from $100 \mu \mathrm{L}$ conjugated beads $(2,500$ beads $/ \mu \mathrm{L})$, followed by the addition of $20 \mu \mathrm{L}$ biotinylated antibody $(0.1 \mu \mathrm{g} / \mathrm{mL})$ and $100 \mu \mathrm{L}$ of 4 -fold diluted sample (or undiluted calibrator) to the

Table 1 Demographic characteristics of the study participants

$\begin{array}{lllllllll}\text { Disease group } & \text { Controls } & \text { Total FTD } & \text { bvFTD } & \text { FTD-MND } & \text { nfvPPA } & \text { svPPA } & \text { IvPPA } & \text { PPA-NOS } \\ \text { No. } & 28 & 67 & 34 & 3 & 13 & 10 & 7 \\ \text { Age, mean (SD), y } & 63.9(7.2) & 64.5(7.9) & 63.0(8.3) & 65.0(0.3) & 67.5(9.7) & 65.2(6.4) & 65.6(5.9) & 63.9(5.2) \\ \text { Male sex, \% } & 46.4 & 61.2 & 73.5 & 66.7 & 23.1 & 60.0 & 71.4 & 71.4 \\ \text { Disease duration, mean (SD), y } & \text { NA } & 5.5(3.7) & 6.2(4.6) & 6.0(4.6) & 3.8(1.5) & 6.0(2.1) & 6.4(2.9) & 4.5(2.5) \\ \text { Serum NfL, mean (SD), pg/mL } & 19.6(8.2) & 77.9(51.3) & 57.8(33.1) & 195.0(69.9) & 82.5(33.8) & 95.9(33.0) & 49.5(19.4) & 91.2(86.6)\end{array}$

Abbreviations: bvFTD = behavioral variant of frontotemporal dementia; FTD = frontotemporal dementia; FTD-MND = frontotemporal dementia with motor neuron disease; IVPPA = logopenic variant of primary progressive aphasia; NA = not applicable; NfL = neurofilament light chain; nfvPPA = nonfluent variant of primary progressive aphasia; PPA-NOS = primary progressive aphasia not otherwise specified; svPPA = semantic variant of primary progressive aphasia. Total FTD does not include IvPPA. 
Figure 1 Serum neurofilament light chain concentrations in participants by (A) clinical diagnosis and (B) genetic status
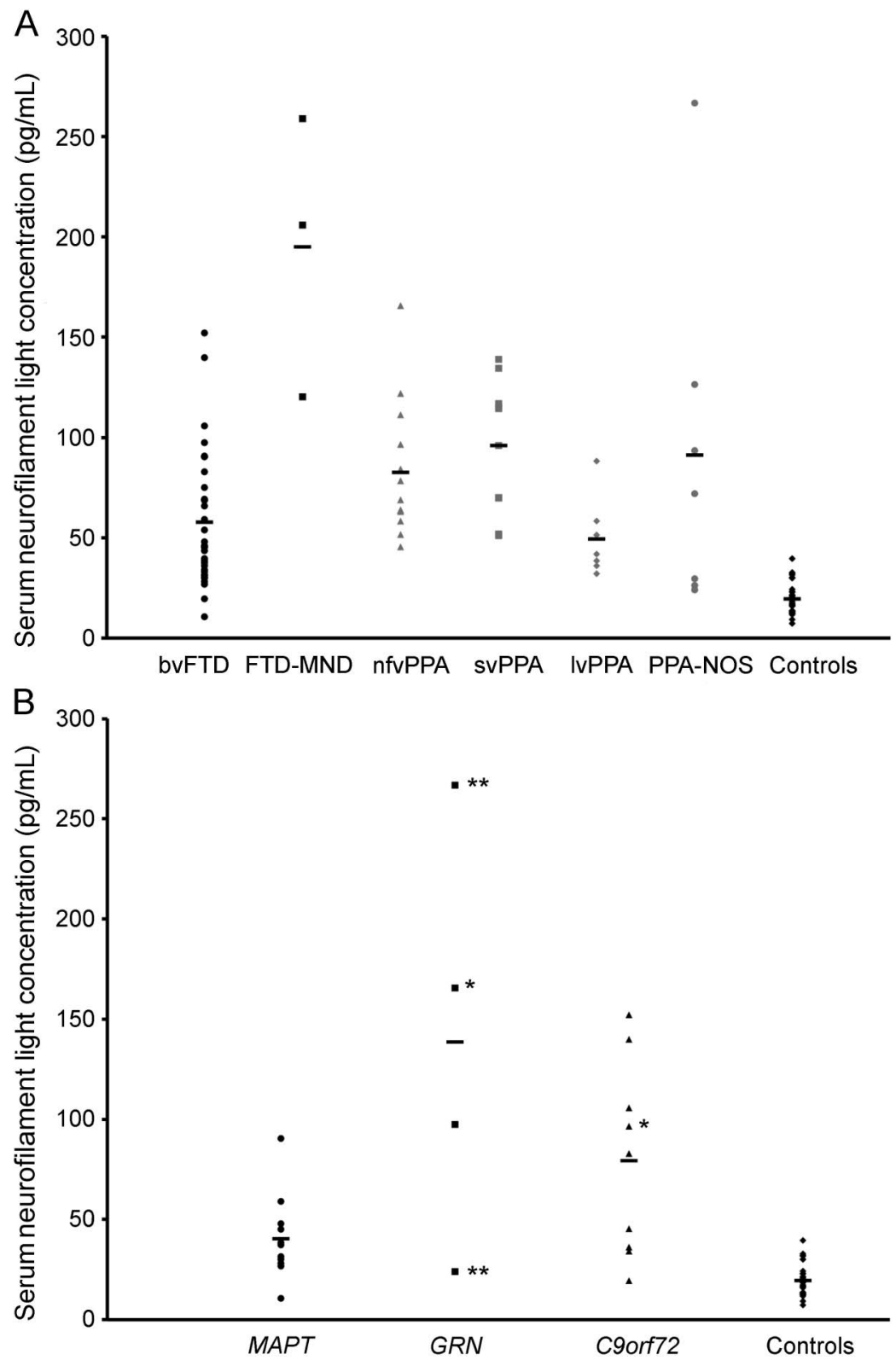

All genetic FTD patients have behavioral variant of frontotemporal dementia (bvFTD) except for those *with nonfluent variant of primary progressive aphasia (nfvPPA) and ** with primary progressive aphasia not otherwise specified (PPA-NOS). FTD-MND = frontotemporal dementia- motor neuron disease; lVPPA = logopenic variant of primary progressive aphasia; svPPA $=$ semantic variant of primary progressive aphasia.

bead pellet. For both samples and calibrator, the same diluent was used (phosphate-buffered saline; $0.1 \%$ Tween-20; $2 \%$ bovine serum albumin; $10 \mu \mathrm{g} / \mathrm{mL}$ TRU Block [Meridian Life Science, Inc, Memphis, TN]). After a 47-cadence incubation (1 cadence $=45$ seconds), the beads were washed, followed by the addition of $100 \mu \mathrm{L}$ streptavidin-conjugated $\beta$-galactosidase $(150 \mathrm{pmol} / \mathrm{L}$, catalog No. 100439, Quanterix). This was followed by a 7 cadence incubation and a wash. Before reading, $25 \mu \mathrm{L}$ resorufin $\beta-\mathrm{D}$-galactopyranoside (catalog No. 100017, Quanterix) was added. The calibrator curve was constructed by use of the standard from the NfL ELISA (NF-Light, UmanDiagnostics) in triplicate. The lower limits of detection and quantification, as defined by the concentration derived from the signal of blank samples (sample diluent) +3 and $10 \mathrm{SD}$, were 0.97 and $2.93 \mathrm{pg} / \mathrm{mL}$, respectively. To evaluate the linearity of the assay, 6 different samples were analyzed at 4 - (default), 8-, and 16-fold dilution, and the average coefficient of variation for the concentration measured at the different dilutions was $11.5 \%$. All samples were measured as duplicates. The mean coefficient of variation of duplicate concentrations was $4.3 \%$. In addition, a quality control sample was measured in duplicate on each of the 7 runs used to complete the study. The intra-assay coefficient of variation for this sample was $<10 \%$. All measurements were performed by board-certified laboratory technicians in one round of experiments using one batch of reagents.

Psychometric assessment. Forty-seven participants had psychometric testing at baseline, usually on the same day as serum sampling but at a maximum of 6 months from the time of sample collection (mean interval 0.0 years [SD 0.2 years]): 22 with bvFTD, 2 with FTD-MND, and 23 with PPA (9 with nfvPPA, 9 with svPPA, and 5 with PPA-NOS). Twenty-nine participants had follow-up psychometric testing at an interval of 1.1 years (SD 0.2 years): 11 with bvFTD, 2 with FTDMND, and 16 with PPA ( 5 with nfvPPA, 7 with svPPA, and 4 with PPA-NOS). Testing included the Wechsler Abbreviated Scale of Intelligence Vocabulary, Block Design, Similarities, and Matrices subtests ${ }^{20}$; the Recognition Memory Tests for Faces and Words ${ }^{21}$; the Graded Naming Test ${ }^{22}$; the Graded Difficulty Calculation $\mathrm{Test}^{23}$; and the Delis-Kaplan Executive Function System Color-Word Interference Test ${ }^{24}$ as well as the Mini-Mental State Examination. ${ }^{25}$

Neuroimaging analysis. Forty-six of the participants with FTD had volumetric T1 brain MRI on a 3T Siemens Trio scanner performed usually on the same day as serum sampling but at a maximum of 6 months from the time of sample collection (mean interval 0.0 years [SD 0.2 years]): 24 with bvFTD, 2 with FTD$\mathrm{MND}$, and 20 with PPA (8 with nfvPPA, 8 with svPPA, 4 with PPA-NOS). Twenty-nine participants had a follow-up scan at 1.1 years (SD 0.4 years) after the baseline scan: 13 with bvFTD, 2 with FTD-MND, and 14 with PPA (5 with nfvPPA, 6 with svPPA, and 3 with PPA-NOS). Whole-brain volumes were measured with a semiautomated segmentation method $^{26}$ with annualized whole-brain atrophy rates calculated with the boundary shift integral. ${ }^{27}$ Individual lobar cortical volumes were measured with a multiatlas segmentation propagation approach following the brainCOLOR protocol (www.braincolor.org), combining regions of interest to calculate gray matter volumes for each lobe. ${ }^{28,29}$ Annualized lobar atrophy rates were calculated using the differences in volumes between the baseline and follow-up scans and dividing by the interval between scans.

Statistical analysis. Serum NfL concentrations were initially compared between the control group and the total FTD group. The Levene test for homogeneity demonstrated unequal variances between these 2 groups (Levene statistic $=22.8 ; p<0.001$ ); therefore, the Welch $t$ test (without assumptions for equal variance) was used to compare the groups. Serum NfL data were normally distributed (Kolmogorov-Smirnov test), so an analysis of variance was used to compare mean serum NfL concentrations across each of the clinical subgroups (bvFTD, FTD-MND, nfvPPA, svPPA, lvPPA, and PPA-NOS) and across the genetic FTD subgroups (MAPT, GRN, and C9orf72), and to compare each of these subgroups with the control group. To allow for unequal variance, the Games-Howell correction was used for post hoc pairwise comparisons between groups. The same statistical methods were also used to compare NfL levels between the genetic subgroups and between each of these groups and the control group. The Pearson correlation coefficient was used to examine the association 


\begin{tabular}{|c|c|c|c|c|c|c|}
\hline \multirow[t]{2}{*}{ Table 2} & \multicolumn{6}{|c|}{ Comparison of serum neurofilament light chain concentrations between the disease subgroups and control group } \\
\hline & bvFTD & FTD-MND & nfvPPA & svPPA & IvPPA & PPA-NOS \\
\hline Controls & $-38.2(5.9)$ & $-175.3(40.4)$ & $-62.9(9.5)$ & $-76.2(10.5)$ & $-29.9(7.5)$ & $-71.6(32.8)$ \\
\hline p & $<0.001$ & 0.185 & $<0.001$ & 0.001 & 0.053 & 0.413 \\
\hline bvFTD & & $-137.2(40.8)$ & $-24.7(10.9)$ & $-38.1(11.9)$ & $8.3(9.3)$ & $-71.6(32.8)$ \\
\hline$p$ & & 0.276 & 0.308 & 0.070 & 0.968 & 0.413 \\
\hline FTD-MND & & & $112.4(41.5)$ & 99.1 (41.7) & $145.4(41.0)$ & $103.8(51.9)$ \\
\hline$p$ & & & 0.374 & 0.451 & 0.248 & 0.509 \\
\hline nfvPPA & & & & $-13.3(14.0)$ & 33.0 (11.9) & $-8.7(34.0)$ \\
\hline$p$ & & & & 0.959 & 0.136 & 1.000 \\
\hline svPPA & & & & & $46.3(12.7)$ & 4.7 (34.4) \\
\hline$p$ & & & & & 0.032 & 1.000 \\
\hline IvPPA & & & & & & -41.7 (33.5) \\
\hline$p$ & & & & & & 0.857 \\
\hline
\end{tabular}

Abbreviations: bvFTD = behavioral variant of frontotemporal dementia; FTD-MND = frontotemporal dementia with motor neuron disease; IVPPA = logopenic variant of primary progressive aphasia; nfvPPA = nonfluent variant of primary progressive aphasia; PPA-NOS = primary progressive aphasia not otherwise specified; svPPA = semantic variant of primary progressive aphasia.

Values are given as mean difference in serum neurofilament concentration between groups (SEM) and refer to comparison of rows vs columns.

between serum NfL concentrations and each of the cognitive and imaging measures (with a Bonferroni correction for multiple comparisons also assessed, i.e., $p<0.005$ for the cognitive measures and $p<0.007$ for the imaging measures).

RESULTS Serum NfL concentrations in the control and total FTD groups and in each clinical subgroup are shown in table 1 . The lowest serum NfL concentration in the study $(7.2 \mathrm{pg} / \mathrm{mL})$ was well above the lower limits of detection and quantification of the assay. Serum NfL concentrations were significantly higher in the total FTD group vs controls (mean $77.9 \mathrm{pg} / \mathrm{mL}[\mathrm{SD} 51.3 \mathrm{pg} / \mathrm{mL}]$ and $19.6 \mathrm{pg} / \mathrm{mL}[\mathrm{SD}$ $8.2 \mathrm{pg} / \mathrm{mL}$ respectively; mean difference $=58.3 \mathrm{pg} / \mathrm{mL}$, $95 \%$ confidence interval 45.4-71.1; $p<0.001)$. In distinguishing FTD from controls, a cutoff of $33 \mathrm{pg} / \mathrm{mL}$ gave a sensitivity of $84 \%$ and specificity of $96 \%$. Serum $\mathrm{NfL}$ concentrations were also significantly higher in the majority of the clinical FTD subgroups compared with the control group (Welch statistic $=25.1, d f 5,13.2$; $p<0.001$ ) (figure 1A, table 2). Compared with controls, serum NfL concentrations were higher in patients with bvFTD, nfvPPA, and svPPA. Although patients with FTD-MND had higher mean serum $\mathrm{NfL}$ concentrations than controls (and all of the other groups), this difference did not reach statistical significance, likely because of the small sample size of the FTD-MND group. Serum NfL concentrations did not differ significantly between any of the clinical FTD subgroups, although there was a (not significant) trend toward a higher level in patients with svPPA compared with patients with bvFTD (mean difference $=38.1$, $p=0.070$ ). There was a significantly higher level in patients with svPPA compared with lvPPA (mean difference $=46.3, p=0.032$ ).
Mean NfL concentrations were higher than controls in each of the genetic subgroups (figure $1 \mathrm{~B}$, table 3): $138.5 \mathrm{pg} / \mathrm{mL}(\mathrm{SD} 103.3 \mathrm{pg} / \mathrm{mL}$ ) in $G R N, 79.2$ $\mathrm{pg} / \mathrm{mL}$ (SD $48.2 \mathrm{pg} / \mathrm{mL}$ ) in C9orf 7 , and $40.5 \mathrm{pg} /$ $\mathrm{mL}$ (SD $20.9 \mathrm{pg} / \mathrm{mL}$ ) in MAPT mutations. However, only the MAPT subgroup (mean difference from controls $=20.8,95 \%$ confidence interval $=1.4-40.3, p$ $=0.035$ ) and the C9orf72 subgroup (mean difference from controls $=59.5,95 \%$ confidence interval $=$ 8.0-111.0, $p=0.025$ ) were significantly different, with the lack of difference in the GRN subgroup likely due to small sample size (table 3 ). Despite the apparent larger mean NfL levels in GRN and C9orf72 compared with MAPT mutations, there was no significant difference in levels between the genetic subgroups (table 3).

Baseline and longitudinal cognitive and imaging measures are shown in table 4. Serum NfL concentrations

\begin{tabular}{|clll|}
\hline Table 3 & \multicolumn{3}{l|}{$\begin{array}{l}\text { Comparison of serum neurofilament } \\
\text { light chain concentrations between the } \\
\text { genetic subgroups and control group }\end{array}$} \\
& MAPT & GRN & C9orf72 \\
Control & $-20.8(6.5)$ & $-118.8(51.7)$ & $-59.5(16.2)$ \\
p & 0.035 & 0.277 & 0.025 \\
MAPT & & $-98.0(52.0)$ & $-38.7(17.3)$ \\
p & & 0.386 & 0.175 \\
GRN & & & $59.3(54.1)$ \\
p & & & 0.712 \\
\hline
\end{tabular}

Values are given as mean difference in serum neurofilament concentration between groups (SEM) and refer to comparison of rows vs columns. 
Table 4 Cognitive and imaging characteristics of the frontotemporal dementia study participants

Baseline, mean (SD) Longitudinal, mean (SD)

\begin{tabular}{lll} 
Cognitive measures & & \\
Participants, $\mathbf{n}$ & 47 & $-1.7(5.0)$ \\
MMSE & $23.8(5.7)$ & $-0.9(2.9)$ \\
WASI vocabulary & $4.4(4.4)$ & $-0.4(2.6)$ \\
WASI block design & $8.7(4.3)$ & $-1.5(3.1)$ \\
WASI similarities & $5.9(4.3)$ & $0.1(2.6)$ \\
WASI matrices & $9.4(4.3)$ & $-0.9(3.7)$ \\
RMT faces & $5.2(4.1)$ & $-1.5(4.1)$ \\
RMT words & $6.1(4.5)$ & $-1.7(3.0)$ \\
Graded naming test & $4.2(4.4)$ & $-0.9(2.4)$ \\
Graded difficulty calculation test & $7.8(5.0)$ & $-1.7(2.5)$ \\
D-KEFS Color-Word Interference Test & $6.1(5.1)$ & \\
Imaging measures & & 29 \\
Participants, $\boldsymbol{n}$ & 46 & $1.9(1.5)$ \\
Whole brain & $72.6(5.0)$ & $2.2(2.7)$ \\
Frontal & $10.4(1.0)$ & $2.7(2.4)$ \\
Temporal & $7.0(0.9)$ & $1.2(2.9)$ \\
Parietal & $6.0(0.5)$ & $0.7(2.5)$ \\
Occipital & $4.9(0.4)$ & $2.6(2.6)$ \\
Insula & $0.8(0.1)$ & $1.2(2.0)$ \\
Cingulate & $1.6(0.1)$ & \\
\hline & & \\
\hline
\end{tabular}

Abbreviations: D-KEFS $=$ Delis-Kaplan Executive Function System; MMSE $=$ Mini-Mental State Examination; RMT = Recognition Memory Test; WASI = Wechsler Abbreviated Scale of Intelligence.

Baseline cognitive measures are standard scores except for the MMSE (out of 30). Longitudinal cognitive scores are annualized change in standard score (or change in MMSE score); a negative score is a decrease in score. Baseline brain volumes are expressed as a percentage of total intracranial volume (measured in Statistical Parameter Mapping [SPM12]). Longitudinal imaging measures are annualized rates of atrophy (\%).

correlated with baseline measures of executive dysfunction (Wechsler Abbreviated Scale of Intelligence similarities $[r=-0.32, p=0.03]$ and Delis-Kaplan Executive Function System Color-Word Interference ink color naming task $[r=-0.35, p=0.03])$ but not with other baseline psychometric tests or with longitudinal changes in psychometric measures. However, no cognitive measures survived correction for multiple comparisons. There were also no significant correlations with baseline brain volumes. However, serum NfL levels were correlated with rates of whole brain $(r=0.46, p=0.01)$, frontal lobe $(r=0.53, p=0.003$; figure 2$)$, and parietal lobe $(r=$ $0.38, p=0.04$ ) atrophy, although not with other lobar atrophy rates. Only the correlation with frontal lobe atrophy rate survived correction for multiple comparisons.

DISCUSSION Using an ultrasensitive immunoassay, we show that serum NfL concentrations are raised in FTD and that higher concentrations are associated with faster rates of brain atrophy. These findings suggest that serum NfL concentrations reflect the intensity of the disease in FTD and that higher concentrations are associated with a more rapid disease progression. Within the FTD subtypes, there was a tendency for groups with probable TDP-43 pathology (svPPA and FTD-MND clinically, $G R N$ and C9orf 72 mutations genetically) to have raised levels compared with those associated with tau pathology (MAPT mutations), although within all groups there is substantial variability. With a lower limit of quantification of $0.26 \mathrm{pg} / \mathrm{mL}$, all samples, including those from normal controls, could be reliably quantified, which is an advantage over earlier studies on serum NfL in other conditions. ${ }^{14,30-32}$

The results of this study are consistent with those found in previous CSF studies of NfL concentrations in FTD: levels are consistently higher in patients with FTD $^{4-11}$ and tend to be increased in those with probable TDP-43 pathology. ${ }^{10,11}$ Certainly for genetic FTD, for which GRN and C9orf 72 mutations are associated with TDP-43 pathology, this is consistent with the more rapid progression (and shorter disease duration) seen in many patients within these 2 mutation groups (independent of clinical syndrome) compared with the relatively slower progression of patients with MAPT mutations (which is associated with tau pathology). ${ }^{33}$ One previous study also suggested a correlation of CSF NfL with measures of disease severity and, consistent with our study, showed an association of levels with frontal lobe atrophy. ${ }^{11}$

We found that serum NfL levels were correlated with the rate of subsequent brain atrophy but not with the baseline brain volumes. Measures of brain atrophy are likely to be better measures of the disease intensity than just a single cross-sectional measure of the whole-brain or lobar volumes, which reflect disease duration and normal variation as well as disease activity. Serum NfL levels correlated with baseline measures of executive function but not with longitudinal measures. A number of the patients had scored at near the floor on executive tasks at baseline; therefore, there is less ability to measure progression with such measures when assessed longitudinally.

It will be important to investigate patients at different stages of the disease because this may influence the association between NfL and rates of atrophy. The Genetic Frontotemporal Dementia Initiative (GENFI) study (www.genfi.org.uk) has recently shown that pathologic rates of brain atrophy appear to start up to 10 years before symptom onset but are variable between different genetic mutations. ${ }^{29}$ If serum NfL concentrations represent measures of disease intensity, then we would predict that levels would start to increase around 10 years before onset and may provide a useful noninvasive marker of proximity to symptom onset.

There are a number of limitations to this study. In particular, the majority of the patients did not have 
Figure 2 Relationship of serum neurofilament light chain (NfL) concentrations to frontal lobe atrophy rate

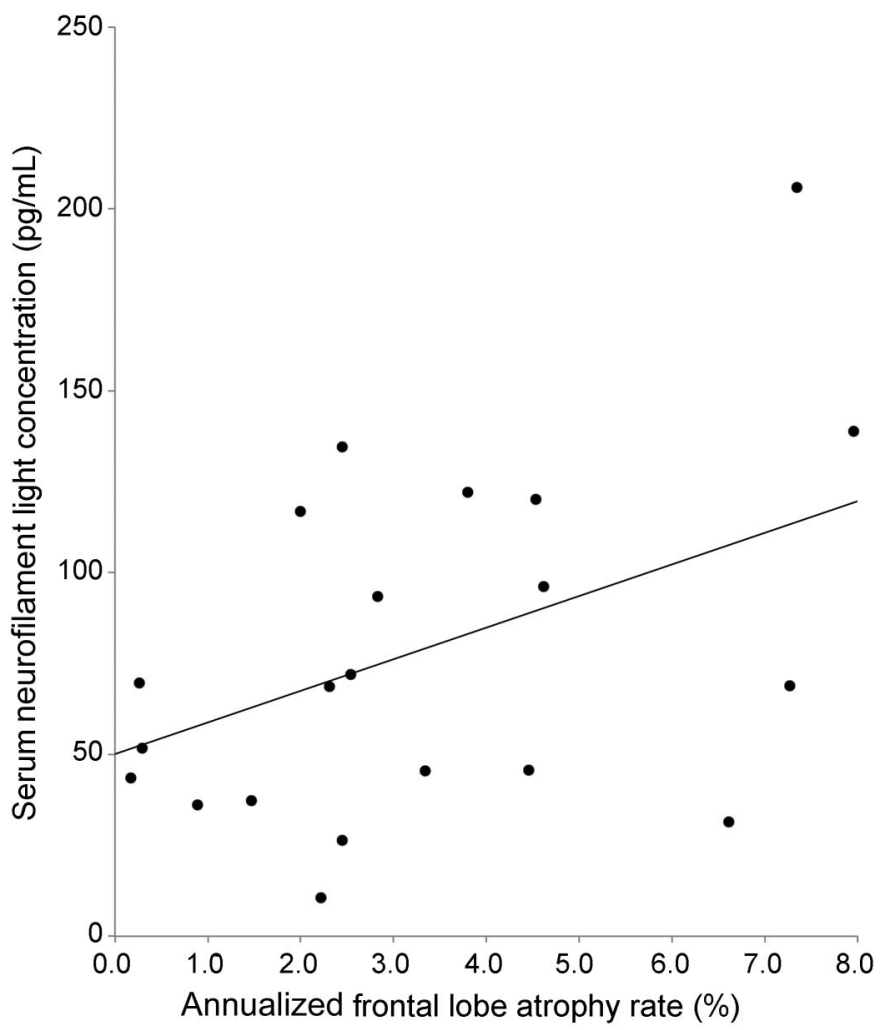

Serum NfL concentrations are correlated with frontal lobe atrophy rates $(r=0.53, p=$ 0.003). Points indicate individual patient values, and the straight line indicates the line of best fit from a linear regression model of serum $\mathrm{NfL}$ on annualized frontal lobe atrophy rate.

pathologic confirmation of the cause of their illness, and future studies should investigate serum NfL levels in different FTD pathologies. Although there is a relatively large number of cases for a study of a rare disorder like FTD, the individual numbers are small in each subgroup (particularly the FTD-MND group), and it would be useful for future studies to investigate larger groups of the individual clinical and genetic subtypes. Clinical measures of disease staging in FTD have only recently been designed (such as the Frontotemporal Lobar Degeneration-Clinical Dementia Rating ${ }^{34}$ and Frontotemporal Dementia Rating Scale ${ }^{35}$ ) and were not available in this cohort; it will be important for future studies to compare such measures with serum NfL levels.

Higher serum NfL concentrations are associated with more rapid brain atrophy and may therefore reflect disease intensity in FTD. Because blood sampling is less invasive and has better patient acceptability than lumbar puncture, serum NfL may provide important prognostic information and prove to be a useful outcome measure for clinical trials in FTD. However, further studies will be required to understand the factors affecting the variability in NfL concentration and to determine whether it can be a useful measure within individual patients.

\section{AUTHOR CONTRIBUTIONS}

J.D.R., K.D.M., E.B., S.M., J.M.S., N.C.F., and J.D.W. were involved in patient recruitment and collection of data. J.T., R.D., N.N., U.A., K.B., and H.Z. were involved in assay development, sample processing, and analysis. J.D.R., I.O.C.W., K.M.D., E.B., E.G., A.F., M.J.C., and S.O. were involved in analysis of the psychometric and imaging data. J.D.R. and I.O.C.W. drafted the initial version and figures. J.D.R., I.O.C.W., and J.M.N. performed the statistical analysis. All authors contributed to reviewing and editing the manuscript.

\section{STUDY FUNDING}

This work was funded by the Medical Research Council, UK, Alzheimer's Research UK, the Vinnova Foundation Sweden, the Torsten Söderberg Foundation at the Royal Swedish Academy of Sciences, the Swedish Research Council, and the Knut and Alice Wallenberg Foundation. The authors acknowledge the support of the NIHR Queen Square Dementia Biomedical Research Unit, Leonard Wolfson Experimental Neurology Centre, and the University College London Hospitals NHS Trust Biomedical Research Centre. The Dementia Research Centre is an Alzheimer's Research UK coordinating center and has received equipment funded by Alzheimer's Research UK and Brain Research Trust. The National Prion Monitoring Cohort study (from which health control serum samples were used) was funded by the Department of Health (England), the National Institute of Health Research's Biomedical Research Centre at UCLH, and the Medical Research Council.

\section{DISCLOSURE}

J. Rohrer is an MRC Clinician Scientist and has received funding from the NIHR Rare Disease Translational Research Collaboration. I. Woollacott is supported by an MRC Clinical Research Training Fellowship (MR/M018288/1). K. Dick, E. Brotherhood, E. Gordon, A. Fellows, J. Toombs, R. Druyeh, J. Cardoso, S. Ourselin, and J. Nicholas report no disclosures relevant to the manuscript. N. Norgren is employed by UmanDiagnostics. He is a cofounder of Brain Biomarker Solutions in Gothenburg AB, a GU Holding-based platform company at the University of Gothenburg. S. Mead and U. Andreasson report no disclosures relevant to the manuscript. K. Blennow is a cofounder of Brain Biomarker Solutions in Gothenburg AB, a GU Holding-based platform company at the University of Gothenburg. J. Schott reports no disclosures relevant to the manuscript. N. Fox is an NIHR Senior Investigator. J. Warren is supported by a Wellcome Trust Senior Clinical Fellowship (091673/Z/10/Z). $\mathrm{H}$. Zetterberg is a cofounder of Brain Biomarker Solutions in Gothenburg $\mathrm{AB}$, a GU Holding-based platform company at the University of Gothenburg. Go to Neurology.org for full disclosures.

Received March 5, 2016. Accepted in final form June 6, 2016.

\section{REFERENCES}

1. Seelaar H, Rohrer JD, Pijnenburg YA, Fox NC, van Swieten JC. Clinical, genetic and pathological heterogeneity of frontotemporal dementia: a review. J Neurol Neurosurg Psychiatry 2011;82:476-486.

2. Warren JD, Rohrer JD, Rossor MN. Clinical review: frontotemporal dementia. BMJ 2013;347:f4827.

3. Rohrer JD, Zetterberg H. Biomarkers in frontotemporal dementia. Biomark Med 2014;8:519-521.

4. Rosengren LE, Karlsson JE, Sjögren M, Blennow K, Wallin A. Neurofilament protein levels in CSF are increased in dementia. Neurology 1999;52:1090-1093.

5. Sjögren M, Rosengren L, Minthon L, Davidsson P, Blennow K, Wallin A. Cytoskeleton proteins in CSF distinguish frontotemporal dementia from AD. Neurology 2000; 54:1960-1964.

6. Brettschneider J, Petzold A, Schottle D, Claus A, Riepe M, Tumani $\mathrm{H}$. The neurofilament heavy chain $(\mathrm{NfH})$ in the cerebrospinal fluid diagnosis of Alzheimer's disease. Dement Geriatr Cogn Disord 2006;21:291-295. 
7. Petzold A, Keir G, Warren J, Fox N, Rossor MN. A systematic review and meta-analysis of CSF neurofilament protein levels as biomarkers in dementia. Neurodegener Dis 2007;4:185-194.

8. Pijnenburg YA, Janssen JC, Schoonenboom NS, et al. CSF neurofilaments in frontotemporal dementia compared with early onset Alzheimer's disease and controls Dement Geriatr Cogn Disord 2007;23:225-230.

9. de Jong D, Jansen RW, Pijnenburg YA, et al. CSF neurofilament proteins in the differential diagnosis of dementia. J Neurol Neurosurg Psychiatry 2007;78:936-938.

10. Landqvist Waldö M, Frizell Santillo A, Passant U, et al. Cerebrospinal fluid neurofilament light chain protein levels in subtypes of frontotemporal dementia. BMC Neurol 2013;13:54.

11. Scherling CS, Hall T, Berisha F, et al. Cerebrospinal fluid neurofilament concentration reflects disease severity in frontotemporal degeneration. Ann Neurol 2014;75:116-126.

12. Teunissen CE, Dijkstra C, Polman C. Biological markers in CSF and blood for axonal degeneration in multiple sclerosis. Lancet Neurol 2005;4:32-41.

\section{Comment: "If you can't measure it, you can't improve it" (Lord Kelvin)}

In clinical practice, disability and its progression are notoriously difficult to quantify, urging the need for reliable soluble biomarkers for neuroaxonal damage. Neurofilament light chain (NfL) is a scaffolding protein of the neural cytoskeleton with important roles in axonal and dendritic branching and growth. After neuronal damage, NfL levels in the CSF increase and thus are considered a highly specific, real-time biomarker of axonal injury.

In the case of serum NfL, the single-molecule array technology substantially improves analytical sensitivity to an extent that measurements in blood-derived samples may be used as surrogate endpoints in neuroprotection trials or even in daily practice in the relatively near future. ${ }^{1,2}$ The ultrasensitive assay used by Rohrer and colleagues ${ }^{1}$ allowed reliable NfL measurements in all serum samples, including healthy controls, a giant stride for a biomarker that is independent of CSF and hence applicable in a routine clinical setting. Moreover, the authors found that their assay is quantitative: higher concentrations were associated with more rapid course of frontal lobe atrophy $(r=0.53, p=0.003) .{ }^{1}$ A strong correlation between CSF and blood NfL levels has been convincingly shown across a number of neurologic diseases, so the lack of CSF data in the current study does not limit the relevance of the findings.

Rohrer and colleagues ${ }^{1}$ provide strong evidence supporting the potential role of serum NfL as a disease progression marker in frontotemporal dementia. Their results are well in line with recent findings in experimental mouse models of proteopathic (tau, $\beta$-amyloid, and $\alpha$-synuclein) neurodegenerative diseases in which NfL levels in CSF and plasma were responsive to experimental manipulation or targeted therapy. ${ }^{3}$ Future studies in large, well-characterized patient cohorts are warranted to further characterize NfL as a primary biomarker to predict and monitor disease progression and to assess treatment responses.

1. Rohrer JD, Woollacott IOC, Dick KM, et al. Serum neurofilament light chain protein is a measure of disease intensity in frontotemporal dementia. Neurology 2016;87: 1329-1336.

2. Kuhle J, Barro C, Andreasson U, et al. Comparison of three analytical platforms for quantification of the neurofilament light chain in blood samples: ELISA, electrochemiluminescence immunoassay and Simoa. Clin Chem Lab Med Epub 2016 Apr 12. doi: $10.1515 / \mathrm{cclm}-2015-1195$

3. Bacioglu M, Maia L, Preische O, et al. Neurofilament light chain in blood and CSF as marker of disease progression in mouse models and in neurodegenerative diseases. Neuron 2016;91:56-66.

Jens Kuble, $M D, P h D$

From Neurology, Departments of Medicine, Clinical Research and Biomedicine, University Hospital Basel, Switzerland.

Study funding: No targeted funding reported.

Disclosure: J.K. reports that the University Hospital Basel as the employer of J.K. has received and dedicated to research support consulting fees from Novartis and Protagen AG; speaker fees from the Swiss MS Society, Biogen, Novartis, Roche, and Genzyme; travel expenses from Merck Serono and Novartis; grants from ECTRIMS Research Fellowship Programme, University of Basel, Swiss MS Society, Swiss National Research Foundation, Bayer (Schweiz) AG, Genzyme, Novartis, and Roche. Go to Neurology.org for full disclosures.
13. Tortelli R, Ruggieri M, Cortese R, et al. Elevated cerebrospinal fluid neurofilament light levels in patients with amyotrophic lateral sclerosis: a possible marker of disease severity and progression. Eur J Neurol 2012;19:1561-1567.

14. Lu CH, Macdonald-Wallis C, Gray E, et al. Neurofilament light chain: a prognostic biomarker in amyotrophic lateral sclerosis. Neurology 2015;84:2247-2257.

15. Rissin DM, Kan CW, Campbell TG, et al. Single-molecule enzyme-linked immunosorbent assay detects serum proteins at subfemtomolar concentrations. Nat Biotechnol 2010;28: 595-599.

16. Kuhle J, Barro C, Andreasson U, et al. Comparison of three analytical platforms for quantification of the neurofilament light chain in blood samples: ELISA, electrochemiluminescence immunoassay and Simoa. Clin Chem Lab Med Epub 2016 Apr 12. doi: 10.1515/cclm-2015-1195.

17. Rascovsky K, Hodges JR, Knopman D, et al. Sensitivity of revised diagnostic criteria for the behavioural variant of frontotemporal dementia. Brain 2011;134:2456-2477.

18. Strong MJ, Grace GM, Freedman M, et al. Consensus criteria for the diagnosis of frontotemporal cognitive and behavioural syndromes in amyotrophic lateral sclerosis. Amyotroph Lateral Scler 2009;10:131-146.

19. Gorno-Tempini ML, Hillis AE, Weintraub S, et al. Classification of primary progressive aphasia and its variants. Neurology 2011;76:1006-1014.

20. Wechsler D. Wechsler Abbreviated Scale of Intelligence. San Antonio, TX: The Psychological Corporation; 1999.

21. Warrington EK. Manual for the Recognition Memory Test for Words and Faces. Windsor, UK: NFER-Nelson; 1984.

22. McKenna P, Warrington EK. Testing for nominal dysphasia. J Neurol Neurosurg Psychiatry 1980;43:781-788.

23. Jackson M, Warrington EK. Arithmetic skills in patients with unilateral cerebral lesions. Cortex 1986;22:611-620.

24. Delis DC, Kaplan E, Kramer JH. Delis-Kaplan Executive Function System (D-kefs). San Antonio, TX: The Psychological Corporation; 2001.

25. Folstein M, Folstein S, McHugh P. The "Mini Mental State": a practical method for grading the cognitive state of patients for the clinician. J Psychiatr Res 1975;12:189-198.

26. Freeborough PA, Fox NC, Kitney RI. Interactive algorithms for the segmentation and quantitation of 3-D MRI brain scans. Comput Methods Programs Biomed 1997;53:15-25.

27. Freeborough PA, Fox NC. The boundary shift integral: an accurate and robust measure of cerebral volume changes from registered repeat MRI. IEEE Trans Med Imaging 1997;16:623-629.

28. Cardoso MJ, Modat M, Wolz R, et al. Geodesic information flows: spatially-variant graphs and their application to segmentation and fusion. IEEE Trans Med Imaging 2015; 34:1976-1988.

29. Rohrer JD, Nicholas JM, Cash DM, et al. Presymptomatic cognitive and neuroanatomical changes in genetic frontotemporal dementia in the Genetic Frontotemporal dementia Initiative (GENFI) study: a cross-sectional analysis. Lancet Neurol 2015;14:253-262.

30. Disanto G, Adiutori R, Dobson R, et al. Serum neurofilament light chain levels are increased in patients with a clinically isolated syndrome. J Neurol Neurosurg Psychiatry 2016;87:126-129.

31. Kuhle J, Gaiottino J, Leppert D, et al. Serum neurofilament light chain is a biomarker of human spinal cord injury 
severity and outcome. J Neurol Neurosurg Psychiatry 2015; 86:273-279.

32. Al Nimer F, Thelin E, Nyström H, et al. Comparative assessment of the prognostic value of biomarkers in traumatic brain injury reveals an independent role for serum levels of neurofilament light. PLoS One 2015;10:e0132177.

33. Mahoney CJ, Beck J, Rohrer JD, et al. Frontotemporal dementia with the C9ORF72 hexanucleotide repeat expansion: clinical, neuroanatomical and neuropathological features. Brain 2012;135:736-750.

34. Knopman DS, Kramer JH, Boeve BF, et al. Development of methodology for conducting clinical trials in frontotemporal lobar degeneration. Brain 2008;131:2957-2968.

35. Mioshi E, Hsieh S, Savage S, Hornberger M, Hodges JR. Clinical staging and disease progression in frontotemporal dementia. Neurology 2010;74:1591-1597.

\section{This Week's Neurology ${ }^{\circledR}$ Podcast}

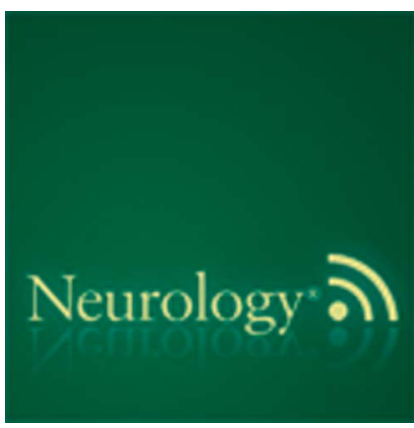

The terrorist inside my husband's brain (see p. 1308)

This podcast begins and closes with Dr. Robert Gross, Editorin-Chief, briefly discussing highlighted articles from the September 27, 2016, issue of Neurology. In the second segment, Dr. Ted Burns talks with Mrs. Susan Schneider Williams about her editorial on learning to deal with her husband's, Robin Williams, Lewy body disease. Dr. Adam Numis reads the e-Pearl of the week about X-linked myopathy with excessive autophagy. In the next part of the podcast, Dr. Andy Schomer concludes his interview with Dr. Eelco Wijdicks on using clinical examination and ancillary tests to come to a conclusion regarding prognosis.

Disclosures can be found at Neurology.org.

At Neurology.org, click on "RSS" in the Neurology Podcast box to listen to the most recent podcast and subscribe to the RSS feed.

CME Opportunity: Listen to this week's Neurology Podcast and earn 0.5 AMA PRA Category $1 \mathrm{CME}$ Credits ${ }^{\mathrm{TM}}$ by answering the multiple-choice questions in the online Podcast quiz.

\section{Share Your Artistic Expressions in Neurology 'Visions'}

AAN members are urged to submit medically or scientifically related artistic images, such as photographs, photomicrographs, and paintings, to the "Visions" section of Neurology ${ }^{\circledR}$. These images are creative in nature, rather than the medically instructive images published in the NeuroImages section. The image or series of up to six images may be black and white or color and must fit into one published journal page. Accompanying description should be 100 words or less; the title should be a maximum of 96 characters including spaces and punctuation.

Learn more at www.aan.com/view/Visions, or upload a Visions submission at submit.neurology.org. 


\section{Neurology}

\section{Serum neurofilament light chain protein is a measure of disease intensity in frontotemporal dementia}

Jonathan D. Rohrer, Ione O.C. Woollacott, Katrina M. Dick, et al. Neurology 2016;87;1329-1336 Published Online before print August 31, 2016

DOI 10.1212/WNL.0000000000003154

This information is current as of August 31, 2016

\section{Updated Information \& Services}

\section{Supplementary Material}

\section{References}

Citations

Subspecialty Collections

Permissions \& Licensing

Reprints including high resolution figures, can be found at: http://n.neurology.org/content/87/13/1329.full

Supplementary material can be found at: http://n.neurology.org/content/suppl/2016/08/31/WNL.0000000000003 154.DC1

http://n.neurology.org/content/supp1/2017/08/07/WNL.0000000000003 154.DC2

This article cites 32 articles, 11 of which you can access for free at: http://n.neurology.org/content/87/13/1329.full\#ref-list-1

This article has been cited by 21 HighWire-hosted articles: http://n.neurology.org/content/87/13/1329.full\#\#otherarticles

This article, along with others on similar topics, appears in the following collection(s):

All Cognitive Disorders/Dementia

http://n.neurology.org/cgi/collection/all_cognitive_disorders_dementia Frontotemporal dementia

http://n.neurology.org/cgi/collection/frontotemporal_dementia

Information about reproducing this article in parts (figures,tables) or in its entirety can be found online at:

http://www.neurology.org/about/about_the_journal\#permissions

Information about ordering reprints can be found online:

http://n.neurology.org/subscribers/advertise

Neurology ${ }^{\circledR}$ is the official journal of the American Academy of Neurology. Published continuously since 1951, it is now a weekly with 48 issues per year. Copyright () 2016 American Academy of Neurology. All rights reserved. Print ISSN: 0028-3878. Online ISSN: 1526-632X.

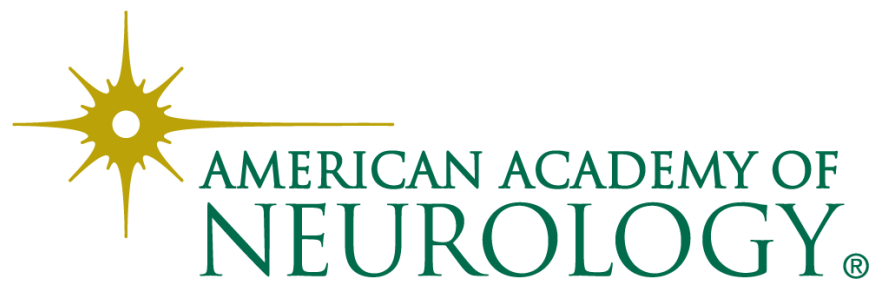

